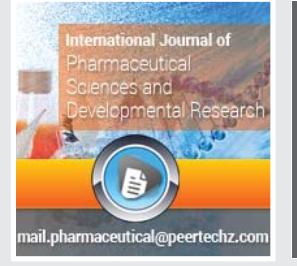

\section{Developing analytical}

\section{parameters and evaluation \\ of thermo fisher urinary}

creatinine enzymatic method

\section{to implement in open system}

chemistry analyzers

\author{
Ashraf Mina ${ }^{1,2 *}$, Leah McNeice ${ }^{1}$, Shanmugam Banukumar ${ }^{1}$ \\ and Santiago Vazquez ${ }^{1}$ \\ ${ }^{1}$ NSW Health Pathology, Forensic \& Analytical Science Service (FASS), Toxicology Unit, Macquarie \\ Hospital, NSW, Australia \\ ${ }^{2}$ Affiliated Senior Clinical Lecturer, Faculty of Medicine and Health, Sydney University, NSW, Australia
}

Received: 21 August, 2020

Accepted: 14 September, 2020

Published: 16 September, 2020

*Corresponding author: Ashraf Mina, NSW Health Pathology, Forensic \& Analytical Science Service (FASS), Toxicology Unit, Macquarie Hospital, PO Box 53, North Ryde Mail Centre, North Ryde NSW 1670, NSW, Australia, Tel: +61 029887 5666, +61 0403108 682; Fax: +61 029805 1259; E-mail: ashraf.mina@health.nsw.gov.au Keywords: Creatinine; Enzymatic creatinine; Sample validity testing; Urinary creatinine

https://www.peertechz.com

\section{Check for updates}

\begin{abstract}
Creatinine assay is used mainly to monitor kidney function and for sample validity when testing for drugs of abuse. Creatinine alkaline picrate method based on Jaffe's reaction calls for extra cleaning procedures to minimize interference from picric acid and sodium hydroxide. Thermo Fisher enzymatic method is more sensitive and specific when compared to alkaline picrate method, but there were no available analytical parameters to setup this enzymatic method on open chemistry analyses platform such as Beckman-Coulter AU5800 analyser. Specific analytical parameters were developed and the assay performance is evaluated against the alkaline picrate method. We successfully set up analytical parameters on Beckman-Coulter AU5800, as an example of open system analyser, and evaluated its performance against Thermo Fisher Jaffe's reaction method. The weighted Deming regression analysis for 408 samples showed a good correlation at a $95 \%$ confidence interval. Slope is 1.065 to 1.091 , $Y$-intercept when $X=0.0$ is -0.9377 to -0.5443 and correlation coefficient ( $r$ ) is 0.993 . The alkaline picrate method Mean and $S D$ for tested samples are 13.2877 and 7.414 respectively and for the enzymatic method are 13.5823 and 7.987 respectively. Many interferences from drugs and other substances are eliminated when using the enzymatic method and the stability of other reagents on-board improved because the reagents used in the enzymatic method are less invasive.
\end{abstract}

\section{Introduction}

Creatinine is a chemical waste product that is produced from normal wear and tear on muscles metabolism and to a smaller extent by eating meat. Healthy kidneys filter creatinine and other waste products from your blood. These waste products are removed from your body through urination. Urinary Creatinine is used to assess the kidney function by measuring the Glomerular Filtration Rate (GFR). Also, urinary creatinine is analysed as part of urine sample validity when testing for drugs of abuse. Urine creatinine can be used as an indicator of urine water content or as a marker identifying a specimen as urine. Greater than normal intake of water will increase the urine water content and lower the creatinine level consequently diluting the amount of drug in urine. Below normal creatinine levels indicate that a person has been drinking excess fluids. Such a reading is a red flag in drug tests because it signifies that the person tested has attempted to tamper with the results by disguising other active by-products that would have otherwise been detected.

No interference from hemolysis, lipemia, or bilirubin detected when using Enzymatic Creatinine methods [1].

The creatinine Jaffe's method (alkaline picrate method) is 
subject to bias due to interfering substances, which means a loss of analytical specificity. Additionally, the Jaffe's method may pose a low risk in selected populations if the estimated Glomerular Filtration Rate (eGFR) results near the $60 \mathrm{ml} /$ min/1.73 $\mathrm{m} 2$ decision limit are interpreted with caution $[2,3]$.

In patients on dialysis, the Jaffe's reaction gave higher creatinine results than the enzymatic test when assays were performed in peritoneal dialysis solutions and pure glucose solutions. It appeared that other components of dialysis solutions, mainly calcium chloride, influenced unpredictably the results of creatinine with the Jaffe's reaction. The specific enzymatic test is a more accurate and reliable method to evaluate creatinine kinetics through the peritoneal membrane when determined in Continuous Ambulatory Peritoneal Dialysis (CAPD) solutions [4].

Albumin, Immunoglobulin G (IgG) and Hemoglobin F ( $\mathrm{Hb} \mathrm{F}$ ) interfered with Jaffe's creatinine assays, leading to inaccuracies in estimated glomerular filtration rates that are clinically important, especially in children and neonates. Because protein error and $\mathrm{Hb} \mathrm{F}$ interference do not occur with any of the enzymatic methods tested, the enzymatic creatinine methods are preferred for evaluation of kidney function in pediatric cases [5]. Both Jaffe and enzymatic methods were found to meet the analytical performance requirements in routine use. However, the enzymatic method was found to have better performance in low creatinine levels [6]. Therefore, using enzymatic creatinine as part of sample validity testing for drugs of abuse will give more reliable results.

The enzymatic method exhibited several advantages over Jaffé-based methods such as smaller sample size, rapid sample throughput, and improved specificity. Glucose, acetoacetate, and cefoxitin did not interfere with the enzymatic method, although bilirubin did cause a negative interference which depended on both creatinine and bilirubin concentrations. The enzymatic method has particular clinical application in neonates, diabetic ketotic patients, and those receiving cephalosporins $[7,8]$. Some cephalosporin-like antibiotics have been shown to interfere with creatinine assays performed by the commonly used Jaffe's methods [9].

The enzymatic creatinine method was better than the picric acid method in terms of specificity and sensitivity [10-14].

\section{Materials and methods}

The reagents used for this evaluation and their part numbers are Thermo Fisher creatinine-detect (CDF1797), Thermo Fisher creatinine calibrator set (CDF100272), Siemens enzyme creatinine-2 (11097533), Siemens chemistry calibrator (11099411) and Bio-Rad Liquicheck urine chemistry control levels 1 (397) and 2 (398). The analyser used is Beckman-Coulter AU5800 from Beckman-Coulter. There were no parameters to set up the Thermo Fisher enzymatic creatinine method on the open system that Beckman-Coulter AU5800 analyser provides.

The author experimented with different instrument settings and different reagents volumes to set up the method and to optimize performance. The performance was verified by testing Bio-Rad QC materials and meeting the targets, analysing external quality control materials using the developed set up and comparing performance with peers using the same method and meeting the targets. The developed analytical parameters of Thermo Fisher urinary creatinine enzymatic method were evaluated and correlated against the established alkaline picrate method. The developed setting on the Beckman-Coulter analyser, as an example of an open system analyser, are summarised in Table 1 . The rest of the other parameters are user-defined.

Table 1: Thermo Fisher enzymatic creatinine method parameter for the BeckmanCoulter AU5800 analyser as an open system analyser.

\begin{tabular}{|c|c|}
\hline Parameter & Value \\
\hline Sample volume & $1.5 \mu \mathrm{L}$ \\
\hline Predilution rate & 15 \\
\hline Diluent bottle & Outside \\
\hline Reagent 1 volume & $90 \mu \mathrm{L}$ \\
\hline Reagent 1 dilution & 0 \\
\hline Reagent 2 volume & $30 \mu \mathrm{L}$ \\
\hline Reagent 2 volume & 0 \\
\hline Primary wavelength & $540 \mathrm{~nm}$ \\
\hline Secondary wavelength & $660 \mathrm{~nm}$ \\
\hline Method & $\mathrm{End}$ \\
\hline Reaction slope & + \\
\hline Measuring point-1 1 & 0 \\
\hline Meast & 27 \\
\hline Measuring point-1 Last & 0 \\
\hline Measuring point-2 Last & 10 \\
\hline Correlation Factor A & 1 \\
\hline Factor for Maker A & 1 \\
\hline
\end{tabular}

\section{Results}

A correlation between Thermo Fisher creatinine alkaline picrate method and Thermo Fisher creatinine enzymatic method was performed using Deming regression analysis. The number of patients' samples analysed is 408. Figure 1 and Table 2 summarise the data analysis.

Correlation coefficient $(r)$ is 0.993 . A value of $r$ greater than 0.7 is considered a strong correlation. Anything between 0.5 and 0.7 is a moderate correlation, and anything less than 0.4 is considered a weak or no correlation

The Thermo Fisher method was accepted according to our laboratory acceptance criteria namely: no big difference in SD, the slope is 1.0 or close to one and correlation coefficient $(r)$ is more than 0.7 .

The Limit of Blank (LoB) represents the highest measurement result that is likely to be observed for an analyte-free sample $(\mathrm{n}=60)$. LoB is $0.01 \mathrm{mg} / \mathrm{dL}(0.7 \mu \mathrm{mol} / \mathrm{l})$. Limit of Detection (LoD) represents the lowest measurable concentration/activity that can be distinguished from zero. It is calculated as the concentration of zero sample $+3 \mathrm{SD}$

Citation: Mina A, McNeice L, Banukumar S, Vazquez S (2020) Developing analytical parameters and evaluation of thermo fisher urinary creatinine enzymatic method to implement in open system chemistry analyzers. Int J Pharm Sci Dev Res 6(1): 030-033. DOI: https://dx.doi.org/10.17352/ijpsdr.000031 
Table 2: Statistical analysis of the data.

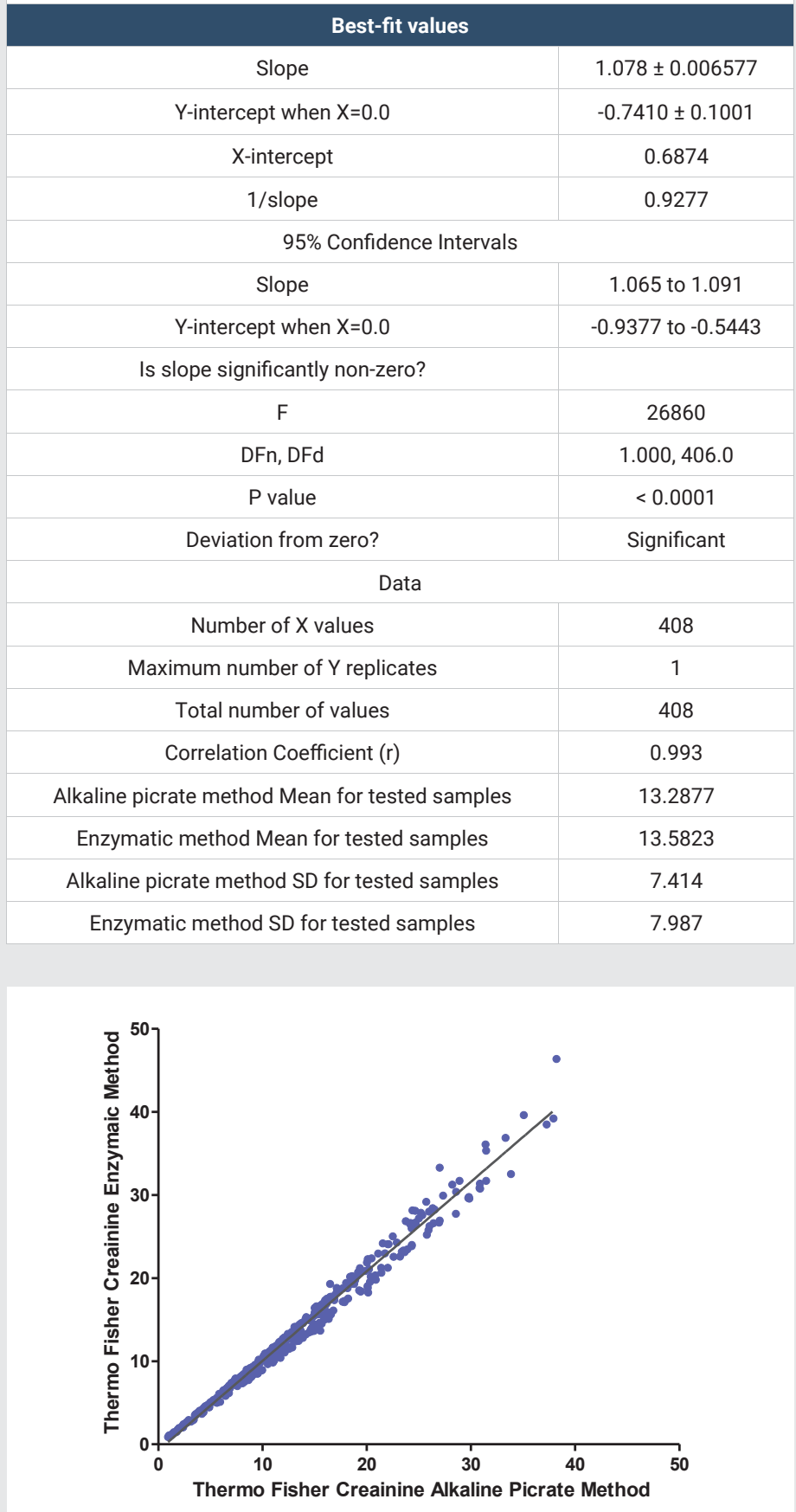

Figure 1: Deming regression analysis between Thermo Fisher creatinine alkaline picrate method and Thermo Fisher creatinine enzymatic method.

(within-run, $\mathrm{n}=24)$. LoD is $0.02 \mathrm{mg} / \mathrm{dL}(0.002 \mathrm{mmol} / \mathrm{L})$ for urine. The Limit of Quantitation (LoO) represents the lowest actual concentration in a sample that can be quantitatively determined $(\mathrm{n}=60)$. LOQ is $0.02 \mathrm{mg} / \mathrm{dL}(0.002 \mathrm{mmol} / \mathrm{L})$ for urine. The imprecision of the creatinine assay for within run and between runs is $\leq 5.0 \%$ Total CV.

The measuring interval for urine is $2.30-452.00 \mathrm{mg} / \mathrm{dL}$ $(0.20-39.96 \mathrm{mmol} / \mathrm{L})$. The measuring interval or the dynamic range of the assay was extended to $6780.00 \mathrm{mg} / \mathrm{dL}(599.36$ $\mathrm{mmol} / \mathrm{L})$. The developed Parameters is set up for the extended measuring interval to avoid multiple sample dilutions beyond the measuring interval.

\section{Discussion}

When it comes to either assessing the renal function or sample validity testing in urine, laboratories must determine the urinary creatinine level. Normal creatinine level indicates the test sample is undiluted, whereas low creatinine level indicates the specimen has either been adulterated or manipulated in some way.

Urinary creatinine clearance in urine and consequently GFR determined by Jaffé assay was much lower than that obtained by the enzymatic method when the serum creatinine concentration was under $2.0 \mathrm{mg} / \mathrm{dl}$ [15]. Creatinine should be reported as "dilute" when the creatinine levels are equal to or greater than $0.1768 \mathrm{mmol} / \mathrm{L}(2 \mathrm{mg} / \mathrm{dL})$ and less than 1.7680 $\mathrm{mmol} / \mathrm{L}(20 \mathrm{mg} / \mathrm{dL})$.

Laboratories testing for drugs of abuse should use creatinine levels as a trigger to conduct further validity testing. If the creatinine level is less than $1.7680 \mathrm{mmol} / \mathrm{L}(20 \mathrm{mg} / \mathrm{dL})$, then the laboratory will also determine the specific gravity level (> 1.0010 but < 1.0030) and $\mathrm{pH}(4.2$ to 9.0). Urine $\mathrm{pH}$ can go up to 9.5 in poor storage condition. $\mathrm{pH}$ is a measure of hydrogen ion concentration, a measure of the acidity or alkalinity of a solution. The specific gravity is an estimate of the concentration of substances dissolved in the solution. If $\mathrm{pH}$ and SG are flagged outside the reference interval, then urine should be tested for the presence of oxidants such as bleach or ammonia, in the urine using Oxidant assay. These procedures are part of the validity testing.

The measurement of creatinine concentrations is an important variable to monitor when attempting to determine if an individual has abstained from marijuana between successive urine specimens. The concentration of the Tetrahydrocannabinol (THC) metabolite can fluctuate from day-to-day depending upon a person's fluid intake. Increased fluid intake will lower both the THC and creatinine concentration in a urine specimen while dehydration will have the opposite effect. If drug testing results are reported as "positive dilute specimen" that is, the specimen tests positive for drugs and is also dilute, the results are considered positive. The fact that the specimen is dilute is irrelevant. If results are reported as "negative dilute specimen" the results are considered negative.

One study claimed that the magnitude of interference with enzymatic assay was greater at a higher creatinine concentration. Therefore, the enzymatic assay might not be appropriate for creatinine measurement in patients using dialysate with dextrose $4.25 \%$ and membrane characteristic of high solute transporter [16]. This study failed to mention which enzymatic method was used. The glucose reference interval in urine is 0 to $0.8 \mathrm{mmol} / \mathrm{L}$. Thermo Fisher package insert for the enzymatic creatinine states that glucose interference was tested at $139 \mathrm{mmol} / \mathrm{L}$ of glucose $(2500 \mathrm{mg} / \mathrm{dl})$ at low and high creatinine concentrations. Targeted creatinine result of 3.7 $\mathrm{mmol} / \mathrm{L}$ gave a result of $5.7 \mathrm{mmol} / \mathrm{L}$ and at a higher targeted creatinine result of $25.5 \mathrm{mmol} / \mathrm{L}$, the obtained result was 24.8 $\mathrm{mmol} / \mathrm{L}$.

Citation: Mina A, McNeice L, Banukumar S, Vazquez S (2020) Developing analytical parameters and evaluation of thermo fisher urinary creatinine enzymatic method to implement in open system chemistry analyzers. Int J Pharm Sci Dev Res 6(1): 030-033. DOI: https://dx.doi.org/10.17352/ijpsdr.000031 
Another study evaluated an automated dry-slide enzymatic method using creatinine iminohydrolase for measurement of creatinine in serum on the Kodak Ektachem analyser found that the enzymatic method had no interference from substances that interfere with Jaffé's methods for creatinine such as acetoacetate. The drugs cephalothin and cephoxitin did not interfere with the enzymatic method, but 5-fluorocytosine interfered significantly with creatinine in the Ektachem method because it causes a colour change similar to that of serum creatinine, resulting in an erroneous detection of serum creatinine. The study concluded that the enzymatic method for creatinine has the speed and precision necessary for routine clinical laboratory use and, except for one drug, the method appears to be more specific for creatinine than Jafee's method [17].

While alkaline picrate and enzymatic creatinine methods had good precision, the enzymatic method had the best precision and was able to detect a biologic change in creatinine more rapidly and consequently detecting early and clinically significant changes in renal function [18].

From practical experience, we found that due to the yellow colour of picric acid reagent used in Jaffe's method, the cleaning of the reaction cuvettes has to be comprehensive to avoid contaminating other assays on-board. Also, the sodium hydroxide used in Jaffe's method can affect the stability of other reagents. Some drugs, such as Cyclosporine were affected and its stability was reduced to only one day when the assay reagents were placed nearby Jaffe's method reagents on the analyser reagents carousels.

\section{Conclusion}

Thermo Fisher enzymatic method is more sensitive and specific when compared to Thermo Fisher alkaline picrate method. We successfully set up analytical parameters on Beckman-Coulter AU5800, as an example of open system analyzers to adopt Thermo Fisher urinary creatinine enzymatic kit and evaluated the developed analytical parameters performance against Jaffe's metod. Many interferences from drugs and other substances are eliminated when using the enzymatic method. Also, the performance and stability of other reagents on-board have improved due to the less invasive chemicals used in the enzymatic method.

\section{References}

1. Badiou S, Dupuy AM, Descomps B, Cristolead JP (2003) Comparison between the enzymatic vitros assay for creatinine determination and three other methods adapted on the olympus analyzer. J Clin Lab Anal 17: 235-240. Link: https://bit.ly/3bZXx3b

2. Schmidt RL, Straseski JA, Raphael KL, Adams AH, Lehman CM (2015) A risk assessment of the jaffe vs enzymatic method for creatinine measurement in an outpatient population. PLoS One 10: e0143205. Link: https://bit.ly/3hqbrwl

3. Boutten A, Bargnoux AS, Carlier MC, Delanaye P, Rozet E, et al. (2013) Enzymatic but not compensated jaffe methods reach the desirable specifications of nkdep at normal levels of creatinine. Results of the french multicentric evaluation. Clin Chim Acta 419: 132-135. Link: https://bit.ly/3mgWctH

4. Larpent $L$, Verger $C$ (1990) The need for using an enzymatic colorimetric assay in creatinine determination of peritoneal dialysis solutions. Perit Dial Int 10 89-92. Link: https://bit.ly/3ixwr5V

5. Cobbaert CM, Baadenhuijsen H, Weykamp CW (2009) Prime time for enzymatic creatinine methods in pediatrics. Clin Chem 55: 549-558. Link: https://bit.ly/3hyErCt

6. Küme T, Sağlam B, Ergon C, Sisman AR (2018) Evaluation and comparison of abbott jaffe and enzymatic creatinine methods: Could the old method meet the new requirements? J Clin Lab Anal 32: e22168. Link: https://bit.ly/33x1acW

7. Crocker H, Shephard MD, White GH (1988) Evaluation of an enzymatic method for determining creatinine in plasma. J Clin Pathol 41: 576-581. Link: https://bit.ly/3mldB4k

8. Goren MP, Osborne S, Wright RK (1986) A peroxidase-coupled kinetic enzymatic procedure evaluated for measuring serum and urinary creatinine. Clin Chem 32: 548-551. Link: https://bit.ly/32v20fT

9. Kroll MH, Koch TR, Drusano GL, Warren JW (1984) Lack of interference with creatinine assays by four cephalosporin-like antibiotics. Am J Clin Pathol 82 214-216. Link: https://bit.ly/3huiQLy

10. Qiu L, Guo X, Zhu Y, Shou W, Gong M, et al. (2013) Effect of picric acid and enzymatic creatinine on the efficiency of the glomerular filtration rate predicator formula. Clin Lab 59: 511-522. Link: https://bit.ly/35ynNk5

11. Harmoinen AP (1996) Bilirubin and metamizol do not interfere with the randox enzymatic creatinine test. An evaluation of a new enzymatic creatinine determination method. Eur J Clin Chem Clin Biochem 34: 975-976. Link: https://bit.ly/35C9wmw

12. Schoenmakers CH, Kuller T, Lindemans J, Blijenberg BG (1993) Automated enzymatic methods for creatinine measurement with special attention to bilirubin interference. Eur J Clin Chem Clin Biochem 31: 861-868. Link: https://bit.ly/32xMZ8x

13. Lindbäck B, Bergman A (1989) A new commercial method for the enzymatic determination of creatinine in serum and urine evaluated: Comparison with a kinetic jaffé method and isotope dilution-mass spectrometry. Clin Chem 35 835-837. Link: https://bit.ly/3icXc9m

14. Guder WG, Hoffmann GE, Hubbuch A, Poppe WA, Siedel J, et al. (1986) Multicentre evaluation of an enzymatic method for creatinine determination using a sensitive colour reagent. J Clin Chem Clin Biochem 24: 889-902. Link: https://bit.ly/3kieOYf

15. Horio $M$, Orita $Y$ (1996) Comparison of jaffé rate assay and enzymatic method for the measurement of creatinine clearance. Nihon Jinzo Gakkai Shi 38: 296299. Link: https://bit.ly/3mINYQN

16. Kaitwatcharachai C, Kaitwatcharachai S, Aeden J, Wiriyasombat D (2011) The glucose interference in creatinine measurement using an enzymatic method: Effect of creatinine concentrations. J Med Assoc Thai 94 Suppl 4: S131-S134 Link: https://bit.ly/3c3oxif

17. Toffaletti J, Blosser N, Hall T, Smith S, Tompkins D (1983) An automated dryslide enzymatic method evaluated for measurement of creatinine in serum. Clin Chem 29: 684-687. Link: https://bit.ly/2Fn3n2Z

18. Toffaletti JG, Hammett-Stabler CA, Gearhart M, Roy Choudhury K, Handel EA (2016) The analytical change in plasma creatinine that constitutes a biologic/ physiologic change. Clin Chim Acta 459: 79-83. Link: https://bit.ly/3bYYFE6

Copyright: (c) 2020 Mina A, et al. This is an open-access article distributed under the terms of the Creative Commons Attribution License, which permits unrestricted use, distribution, and reproduction in any medium, provided the original author and source are credited.

Citation: Mina A, McNeice L, Banukumar S, Vazquez S (2020) Developing analytical parameters and evaluation of thermo fisher urinary creatinine enzymatic method to implement in open system chemistry analyzers. Int J Pharm Sci Dev Res 6(1): 030-033. DOI: https://dx.doi.org/10.17352/ijpsdr.000031 